\title{
POLYNOMIAL IDENTITIES OF INCIDENCE ALGEBRAS
}

\author{
ROBERT B. FEINBERG ${ }^{1}$
}

\begin{abstract}
In this paper we determine the polynomial identities satisfied by incidence algebras. One of our results is logically equivalent to the AmitsurLevitzki Theorem on the polynomial identities satisfied by $K_{n}$, the algebra of of $n \times n$ matrices over a field $K$.
\end{abstract}

0. Preliminaries. The incidence algebra of a finite quasi-ordered set of cardinality $n$ has a natural embedding in $K_{n}$, the algebra of $n \times n$ matrices over the ground field $K$. The polynomial identities (P.I.) satisfied by $K_{n}$ have been determined by the famous theorem of Amitsur and Levitzki. In the spirit of generalization it is thus natural to consider the P.I. satisfied by incidence algebras. We do this here, obtaining a result which is in fact logically equivalent to the Amitsur-Levitzki Theorem.

Let $Q$ be a locally finite quasi-ordered (q.o.) set, i.e., $Q$ has a relation $r$ which is reflexive and transitive and for which every segment $[x, y]=\{z \in Q$; $x r z r y\}$ is finite. The incidence algebra $I(Q)$ of $Q$ over a field $K$ is the associative unital algebra of functions $f: Q \times Q \rightarrow K$ with the property that $f(x, y) \neq 0 \Rightarrow x$ r $y$, under the product

$$
f * g(x, y)=\sum_{x r_{z} y} f(x, z) g(z, y) .
$$

The unit $\delta$ of $I(Q)$ is defined by

$$
\begin{aligned}
\delta(x, y)=1 & \text { if } x=y, \\
=0 & \text { if } x \neq y .
\end{aligned}
$$

For $x r y \in Q$, define $\delta_{x y} \in I(Q)$ by

$$
\begin{aligned}
\delta_{x y}(u, v)=1 & \text { if } x=u, y=v, \\
& =0 \quad \text { otherwise, }
\end{aligned}
$$

and set $e_{x}=\delta_{x x}$. Then $\left\{\delta_{x y}\right\}_{x, y}$ is a linearly independent subset of $I(Q)$, and every element of $I(Q)$ may be regarded as a formal linear combination of

\footnotetext{
Received by the editors February 18, 1975.

AMS (MOS) subject classifications (1970). Primary 16A38, 06A10.

Key words and phrases. Chain, incidence algebra, polynomial identity, quasi-ordered set.

1 This paper was prepared while the author was a National Academy of Sciences-National Research Council Postdoctoral Research Associate at the National Bureau of Standards, Washington, D. C. 20234. The results presented are contained in the author's doctoral dissertation written at the University of Wisconsin. The author's research was sponsored in part by the United States Army under Contract No. DA-31-124-ARO-D-462.
} 
possibly infinitely many elements from this set, using suitable topological notions. See $[1, \S 3]$ or $[2, \S 1]$ for more details.

If $Q$ is of finite cardinality $n$, then $I(Q)$ has a natural embedding $\varphi$ in $K_{n}$. This is obtained as follows: assume $Q=\left\{x_{i}\right\}_{i=1}^{n}$, where $x_{i} r x_{j} \Rightarrow i$ $\leqq j$ or $x_{j} r x_{i}$ (such a labelling is always possible by a simple application of Szpilrajn's Lemma). Then to $f \in I(Q)$ we assign the matrix $\varphi f \in K_{n}$ defined by $(\varphi f)_{u v}=f\left(x_{u}, x_{v}\right)$, for $1 \leqq u, v \leqq n$. In the case when $Q$ is the q.o. set in which $x_{i} r x_{j}, 1 \leqq i, j \leqq n$, the embedding $\varphi$ is in fact onto, so that $I(Q)$ $\simeq K_{n}$.

We now review some basic notions and facts on polynomial identities. See [3, Chapter 1] for more details.

Let $A$ be an associative unital algebra over a field $K$. We say that $A$ satisfies a polynomial identity of degree $k$ if $\exists$ a polynomial $h\left(x_{1}, \ldots, x_{k}\right)$ in the $k$ noncommuting indeterminates $x_{1}, \ldots, x_{k}$ such that $h\left(\alpha_{1}, \ldots, \alpha_{k}\right)=0 \forall \alpha_{1}$, $\ldots, \alpha_{k} \in A$. The standard polynomial of degree $k$ is defined by

$$
\left[x_{1}, \ldots, x_{k}\right]=\sum_{\sigma \in S_{k}} \operatorname{sgn} \sigma x_{\sigma 1} \cdots x_{\sigma k},
$$

where $S_{k}$ is the symmetric group on $k$ letters and sgn $\sigma$ denotes the parity of the permutation $\sigma$. The algebra $A$ satisfies the standard polynomial identity of degree $k$, abbreviated S.P.I. $(k)$, if $\left[\alpha_{1}, \ldots, \alpha_{k}\right]=0 \forall \alpha_{1}, \ldots, \alpha_{k} \in A$.

It may be shown that if $A$ satisfies a nontrivial polynomial identity of degree $k$, then $A$ satisfies the polynomial identity $p\left(\alpha_{1}, \ldots, \alpha_{k}\right)=0 \forall \alpha_{1}, \ldots, \alpha_{k}$ $\in A$, where

$$
p\left(x_{1}, \ldots, x_{k}\right)=x_{1} x_{2} \cdots x_{k}+\sum_{\sigma \in S_{k} ; \sigma \neq 1} c(\sigma) x_{\sigma 1} \cdots x_{\sigma k},
$$

for $c(\sigma) \in K$. In addition, the standard polynomial of degree $k$ is multilinear and skew-symmetric in its arguments. Finally, the algebra $K_{n}$ satisfies no P.I. of degree $<2 n$ and satisfies S.P.I. $(2 n)$ (the latter statement being the AmitsurLevitzki Theorem).

1. Results. We now discuss P.I. for incidence algebras.

DEFINITION. A multichain of length $n$ in a quasi-ordered set $Q$ is a sequence of elements $\left\{x_{0}, \ldots, x_{n}\right\}$ of $Q$ such that $x_{i} r x_{i+1}$, for $0 \leqq i \leqq n-1$. A chain of length $n$ is a multichain in which all the elements are distinct.

Our results consist in relating the above concepts to P.I. of incidence algebras.

THEOREM 1. Let $Q$ be a locally finite q.o. set. If $Q$ has a chain of length $n-1$, then $I(Q)$ satisfies no P.I. of degree $<2 n$.

Proof. Let $\left\{z_{0}, z_{1}, \ldots, z_{k-1}\right\}$ be a chain of length $k-1$ in $Q$, where $k \leqq n$. Then

$$
e_{z_{0}} * \delta_{z_{0} z_{1}} * e_{z_{1}} * \cdots * e_{z_{k-2}} * \delta_{z_{k-2} z_{k-1}} * e_{z_{k-1}}
$$

is the product of $2 k-1$ terms of $I(Q)$ with value

$$
\delta_{z_{0} z_{k-1}} \neq 0
$$


But any rearrangement in the order of the factors results in zero product. Thus

$$
p\left(e_{z_{0}}, \delta_{z_{0} z_{1}}, e_{z_{1}}, \ldots, \delta_{z_{k-2} z_{k-1}}, e_{z_{k-1}}\right) \neq 0,
$$

for any polynomial $p$ of the form (*) above. Therefore $\operatorname{span}\left\{e_{z_{0}}, \delta_{z_{0} z_{1}}, \ldots, e_{z_{k-1}}\right\}$ cannot satisfy a P.I. of degree $2 k-1$, for $k \leqq n$. Hence $I(Q)$ can satisfy no P.I. of odd degree $<2 n$.

Consider now

$$
e_{z_{0}} * \delta_{z_{0} z_{1}} * e_{z_{1}} * \cdots * e_{z_{k-2}} * \delta_{z_{k-2} z_{k-1}}
$$

This is the product of $2 k-2$ terms of $I(Q)$ with value

$$
\delta_{z_{0} z_{k-1}} \neq 0 \text {, }
$$

but any rearrangement in the order of the factors results in zero product. Reasoning as above, we obtain that

$$
\operatorname{span}\left\{e_{z_{0}}, \delta_{z_{0} z_{1}}, \ldots, \delta_{z_{k-2} z_{k-1}}\right\}
$$

cannot satisfy a P.I. of degree $2 k-2$, for $k \leqq n$. Hence $I(Q)$ can satisfy no P.I. of even degree $<2 n$. This completes proof of the theorem.

Note that if $Q$ is the q.o. set $\left\{x_{i}\right\}_{i=1}^{n}$, where $x_{i} r x_{j}, 1 \leqq i, j \leqq n$, then $I(Q) \simeq K_{n}$ and $Q$ has (in fact consists of) a chain of length $n-1$. Hence an immediate corollary of Theorem 1 is the above-cited result, that $K_{n}$ satisfies no P.I. of degree $<2 n$.

THEOREM 2. Let $Q$ be locally finite. If all chains of $Q$ have length $\leqq n-1$, then $I(Q)$ satisfies $S . P . I .(2 n)$.

Proof. By our preliminary remarks it suffices to show that

$$
\left[\delta_{x_{1} y_{1}}, \ldots, \delta_{x_{2 n} y_{2 n}}\right]=0
$$

for all choices of

$$
\left\{\delta_{x_{i} y_{i}}\right\}_{i=1}^{2 n} \subseteq I(Q) .
$$

We may assume without loss of generality that $y_{1}=x_{2}, \ldots, y_{2 n-1}=x_{2 n}$, under suitable relabelling if necessary, otherwise all terms of

$$
\left[\delta_{x_{1} y_{1}}, \ldots, \delta_{x_{2 n} y_{2 n}}\right]
$$

are 0 and hence $\left[\delta_{x_{1} y_{1}}, \ldots, \delta_{x_{2 n} y_{2 n}}\right]=0$ automatically. Under this assumption we have $x_{1} r y_{1} r y_{2} r \cdots r y_{2 n}$, so that $\left\{x_{1}, y_{1}, \ldots, y_{2 n}\right\}$ is a multichain of length $2 n$.

Discarding repeats, there exists a chain of length $m \leqq 2 n$, say $\left\{z_{0}, \ldots, z_{m}\right\}$, which contains the same elements as $\left\{x_{1}, y_{1}, \ldots, y_{2 n}\right\}$. By hypothesis, $m$ $\leqq n-1$. Let $Q^{\prime}$ be the quasi-ordered set consisting of the elements of this chain. Then

$$
\left\{\delta_{x_{1} y_{1}}, \ldots, \delta_{x_{2 n} y_{2 n}}\right\} \subseteq I\left(Q^{\prime}\right)
$$


and hence every term of $\left[\delta_{x_{1} y_{1}}, \ldots, \delta_{x_{2 n} y_{2 n}}\right]$ is an element of $I\left(Q^{\prime}\right)$. Since $I\left(Q^{\prime}\right) \subseteq K_{m+1} \subseteq K_{n}$ as noted above, $\stackrel{I}{I}\left(Q^{\prime}\right)$ satisfies S.P.I.(2n). Therefore $\left[\delta_{x_{1} y_{1}}, \ldots, \delta_{x_{2 n} y_{2 n}}\right]=0$ and we conclude that $I(Q)$ satisfies S.P.I.(2n).

Note again that if $Q$ is the q.o. set $\left\{x_{i}\right\}_{i=1}^{n}$, where $x_{i} r x_{j}, 1 \leqq i, j \leqq n$, then $I(Q)=K_{n}$ and all chains of $Q$ have length $\leqq n-1$. Hence an immediate corollary of Theorem 2 is the Amitsur-Levitzki Theorem, that $K_{n}$ satisfies S.P.I.(2n). But since the Amitsur-Levitzki Theorem is used in the proof of Theorem 2, we must conclude that the two theorems are logically equivalent.

\section{REFERENCES}

1. P. Doubilet, G.-C. Rota and R. Stanley, The idea of generating function, Proc. Sixth Berkeley Sympos. on Math. Statist. and Probability, vol. II, Univ. of California Press, Berkeley, Calif., 1972, pp. 267-318.

2. R. B. Feinberg, Characterization of incidence algebras, (in preparation).

3. D. S. Passman, Infinite group rings, Pure and Appl. Math., vol. 6, Dekker, New York, 1971. MR 47 \#3500.

Applied Mathematics Division, National Bureau of Standards, Washington, D.C. 20234

Current address: Department of Mathematics, Clarkson College of Technology, Potsdam, New York 13676 